\title{
Direct observation of the Gouy phase shift in THz impulse ranging
}

\author{
R. W. McGowan, R. A. Cheville, and D. Grischkowskya) \\ School of Electrical and Computer Engineering and Center for Laser and Photonic Research, Oklahoma \\ State University, Stillwater, Oklahoma 74078
}

(Received 2 August 1999; accepted for publication 8 December 1999)

\begin{abstract}
Here we present a direct observation of the Gouy effect through $\mathrm{THz}$ impulse scattering from cylindrical and spherical targets. A $\pi / 2$ Gouy phase shift through a one-axis focus compared to the more common $\pi$ phase shift of the two-axis focus is required to interpret the scattering results using a physical optics model. (C) 2000 American Institute of Physics. [S0003-6951(00)02306-8]
\end{abstract}

The field of generation and detection of freely propagating picosecond pulses of $\mathrm{THz}$ radiation has blossomed in the last decade due to the development of phase sensitive optoelectronic transceivers. Coherent detection of the $\mathrm{THz}$ radiation eliminates detection of the thermal radiation background from the surrounding environment for which $k T \approx 6 \mathrm{THz}$ at $300 \mathrm{~K}$. A key feature of the $\mathrm{THz}$ system is that the receiver detects the time resolved electric field of the propagating pulse, i.e., both amplitude and phase. By utilizing this feature, interactions in the $\mathrm{THz}$ regime are now being studied with higher precision and detail. One emerging area of study is $\mathrm{THz}$ impulse ranging in which time domain scattering signatures are obtained with a tabletop scale ranging

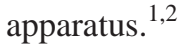

Here we present a direct observation of the Gouy effect through $\mathrm{THz}$ impulse scattering from cylindrical and spherical targets. The Gouy effect is the well known $\pi$ phase shift that any spherical wave front accumulates relative to a uniform plane wave as it propagates through a focus, which also applies to Gaussian beam propagation. ${ }^{3}$ Recently, the importance of the Gouy phase shift to focused single-cycle electromagnetic pulses has been discussed, ${ }^{4}$ and a direct observation of the $\pi$ Gouy phase shift through a spherical focus with $\mathrm{THz}$ pulses was reported. ${ }^{5}$ As presented here, an understanding of the Gouy phase shift through a one-axis (cylindrical) focus of $\pi / 2$ in comparison with a two-axis (spherical) focus of $\pi$ is required to interpret the scattering results using a time domain physical optics (PO) model for scattering. ${ }^{1,2,6}$ The physical optics model allows one to isolate and to understand the different physical mechanisms involved in the scattering process, which are not at all transparent in the often used and well known Mie scattering theory.

The impulse radar range, Fig. 1, consists of an optoelectronic transmitter and receiver along with beam shaping and steering optics. A detailed description of the entire system has previously been published but we shall give a brief one: ${ }^{1}$ $80 \mathrm{fs}$ optical pulses at $820 \mathrm{~nm}$ are focused onto a coplanar stripline on GaAs which is dc biased at $70 \mathrm{~V}$; an electronhole plasma is optically generated and the subsequent acceleration of the carriers generates a near single cycle electromagnetic pulse of $\mathrm{THz}$ radiation. For the spherical target an $f=28 \mathrm{~cm}$ silicon lens (not shown) is placed confocally into

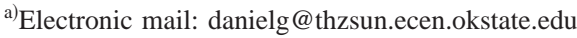

the transmitter arm of the setup. This produces a frequency independent $16 \mathrm{~mm} \mathrm{1/e}$ waist diameter planar wave front at the target position. ${ }^{1}$ For the cylindrical target the beam of $\mathrm{THz}$ pulses is expanded and near planar wave fronts are attained at the far field target $78 \mathrm{~cm}$ from the transmitter. At 1 $\mathrm{THz}$ the deviation from planar phase fronts is only $0.005 \lambda$ over the target diameter of $3 \mathrm{~mm}$. The far field scattered signal is collected (at a bistatic angle of $\theta=11^{\circ}$ for the sphere and $13^{\circ}$ for the cylinder) and focused onto a $30 \mu \mathrm{m}$ dipole antenna on ion-implanted silicon on sapphire. This antenna is photoconductively switched by a second beam with $80 \mathrm{fs}$ pulses, which generates a current that is proportional to the instantaneous electric field of the scattered $\mathrm{THz}$ pulse. By scanning the relative delay between the detected pulse and the gating optical pulse the entire time dependent scattered

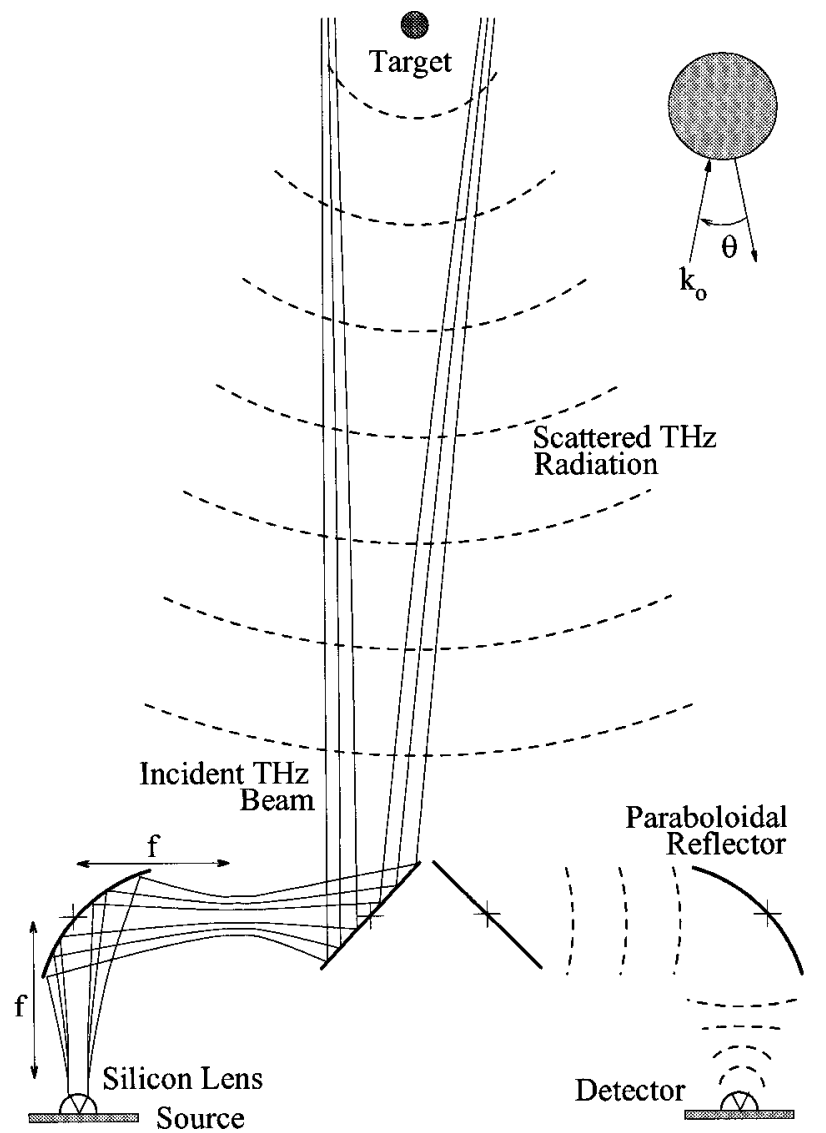

FIG. 1. THz time domain impulse radar range. Scattered radiation collected at a $\theta=13^{\circ}$ bistatic angle. 

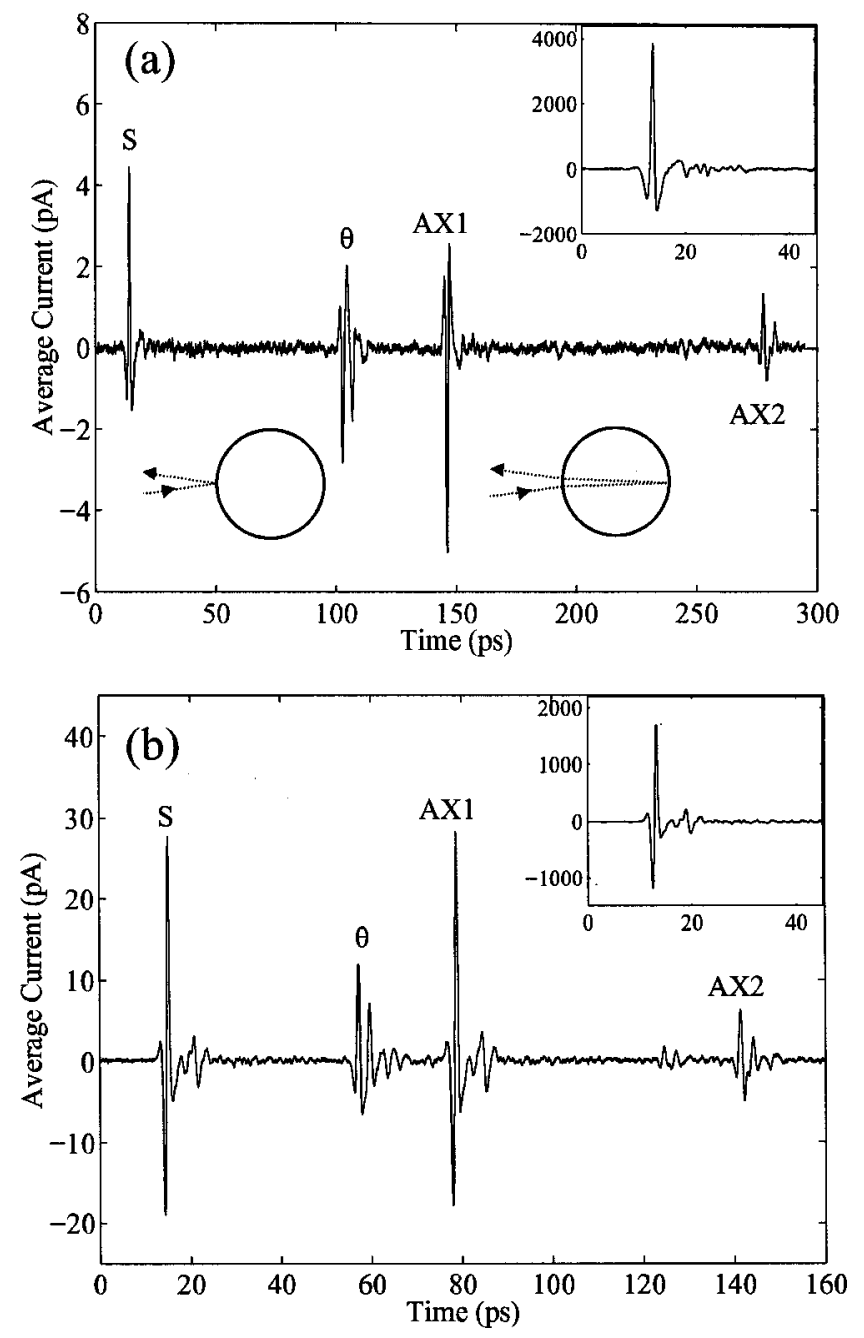

FIG. 2. (a) Measured scattering from a $6.346 \pm 0.001 \mathrm{~mm}$ alumina sphere. (b) Measured scattering from a $3.001 \pm 0.001 \mathrm{~mm}$ diam alumina cylinder. The insets are the reference pulses. Diagrams of the specular reflection $S$ and back axial reflection $A X 1$ are in the inset next to their respective features shown in (a).

pulse is obtained, including both amplitude and phase information.

Both the spherical and the cylindrical targets used in this study are made from aluminum oxide (alumina), which has a $\mathrm{THz}$ index of refraction $n_{a}=3.17$. The spherical target has a diameter of $6.346 \pm 0.001 \mathrm{~mm}$ and the cylindrical target is a $3.001 \pm 0.001 \mathrm{~mm}$ diam, fine-ground cylinder. For both targets the ranging system is first characterized by placing a reference metallic mirror of like shape at the target position; the reference mirrors have a spectrally flat response, yielding accurate measurement of the incident pulse, insets of Figs. 2(a) and 2(b). Utilizing the spherical or cylindrical mirror for the reference pulse provides the same wave front character as the scattered signal from the spherical or cylindrical target, thereby removing any wave front variation effects from the collection and focusing optics of the receiver. ${ }^{2}$ The reference pulses have a frequency bandwidth from 0.1 to $1.5 \mathrm{THz}$ and are polarized in the plane of the bistatic angle, Fig. 1. For the sphere the reference mirror is a $25 \mathrm{~mm}$ diam brass ball and the cylindrical reference is a $30 \mathrm{~mm}$ diam copper tube.

The experimental data for the time domain scattering of the $\mathrm{THz}$ impulse from the alumina sphere consisting of well isolated scattered pulses are shown in Fig. 2, which is an

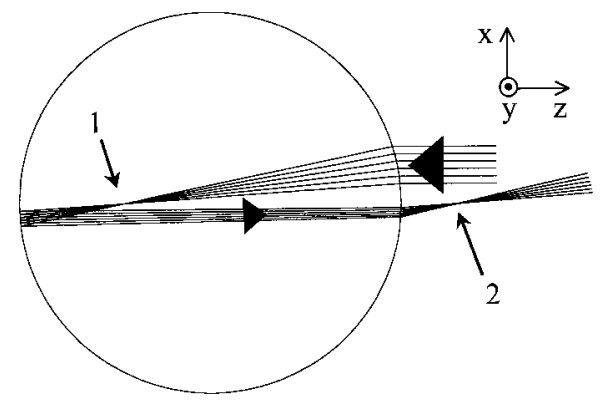

FIG. 3. Ray diagram of scattered back axial reflection $A X 1$ at bistatic angles centered at $\theta=13^{\circ}$ showing the two Gouy phase focal regions.

eight scan average to improve signal to noise. Although not shown here, the data fit exceptionally well with a numerically calculated Mie theory. ${ }^{2,7}$ Since individual features are well resolved, applying the geometrical or $\mathrm{PO}$ model ${ }^{8}$ to the time domain data gives insight into the physical mechanisms involved in scattering. Following this approach three main scattering mechanisms can be distinguished. The pulse labeled $S$ in Fig. 2(a) is the specular reflection from the front surface of the sphere. The pulses labeled $A X 1$ and $A X 2$ are the multiple orders of reflections between the front and back surfaces of the target. The third contributing type is the surface or creeping wave located in time between $S$ and $A X 1$ and labeled $\theta$. Schematic diagrams of $S$ and $A X 1$ are shown inset in Fig. 2(a) next to their respective features. The reference pulse for the sphere is also shown as an inset in Fig. 2(a). A four scan average of the experimental data for the time domain scattering from the alumina cylinder is plotted in Fig. 2(b), where the inset is the reference pulse. It should be noted that the reference pulse and the specular reflection $S$ for both the spherical and cylindrical targets are of identical pulse shape.

As one would expect from the Fresnel equations, there is a $\pi$ phase shift between the scattered signal from the front surface of the sphere $S$ and that from the back surface of the sphere, $A X 1$. This is observed by the inversion of $A X 1$ relative to $S$. However, the scattered signature from the cylinder, which also has an excellent fit with Mie scattering theory, ${ }^{6}$ Fig. 2(b), does not exhibit this same inversion. The back axial scattering, $A X 1$, has the same polarity as the front surface reflection $S$. This $\pi$ phase discrepancy is not explained by Fresnel reflection. In order to accurately apply the PO model the discrepancy must be understood.

The answer is provided by an application of the Gouy phase shift. A cross-sectional ray diagram of the back axial scattered wave $A X 1$ at detection angles centered at $\theta=13^{\circ}$ is shown in Fig. 3. For the $x z$ cross-sectional plane, Fig. 3 applies to both the sphere and the cylinder. As observed, the scattered wave propagates through two focal points, one internal to the target and one external. For the $y z$ plane the sphere also has foci at 1 and 2, whereas the cylinder does not. The back axial reflection, $A X 1$, of the sphere acquires an additional $\pi$ phase shift from each of the two-axis focal points for a total accumulated phase of $\pi+\pi=2 \pi$ from the Gouy effect. Therefore, for the sphere the accumulated phase shift is not observable except as a time shift of the returned pulse. However, in the case of the cylinder, the twodimensional focal symmetry is broken. The focal regions are 

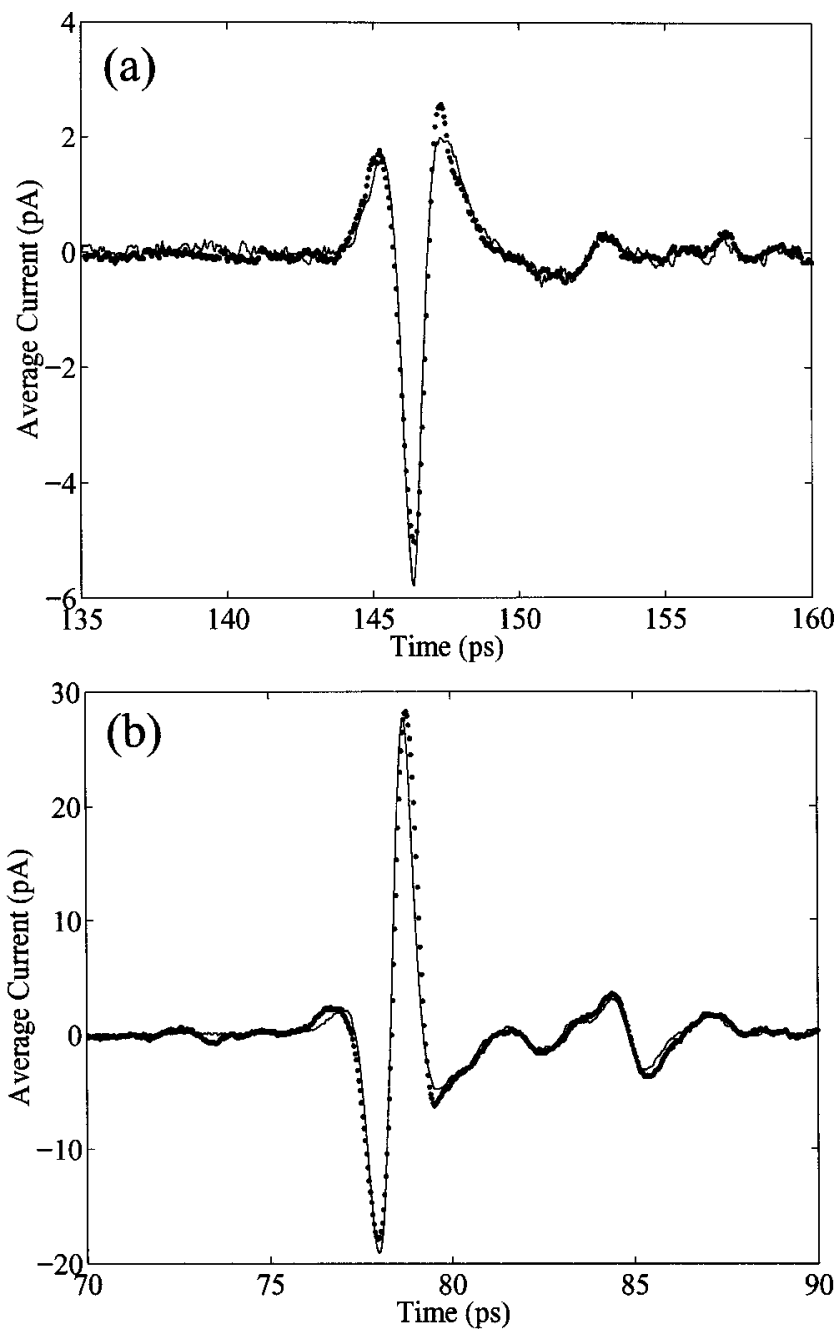

FIG. 4. (a) Back-axial $A X 1$ scattered pulse from the alumina sphere (points) compared with the inverted, scaled specular reflection $S$ overlaid as the solid line. (b) Back-axial $A X 1$ scattered pulse from the alumina cylinder (points) compared with the specular reflection $S$ from the same cylinder overlaid as the solid line.

one dimensional, i.e., only in the $x z$ plane, and form a caustic. Although not relatively well known in optical literature, the accumulated phase shift through a one-axis focus or caustic is $\pi / 2 .{ }^{9}$ The scattered wave $A X 1$ from the cylinder acquires a total accumulated phase shift from the Gouy effect of $\pi / 2+\pi / 2=\pi$, which accounts for the observed inversion of the scattered $A X 1$ between the spherical and cylindrical targets.
The precision and purity of the observed Gouy phase shift between the specular $S$ and the back axial $A X 1$ scattered pulses for the sphere and cylinder are demonstrated by a direct comparison of these pulse shapes, Figs. 4(a) and 4(b), respectively. To fit $A X 1$ for the sphere, $S$ is inverted, scaled in amplitude, and overlaid as the solid line over the point plot of $A X 1$. For the cylinder, $S$ is overlaid as the solid line on the point of $A X 1$ without inversion or scaling, Fig. 4(b). As observed by the excellent fit in both cases, other than the Gouy phase shift, the back axial scattered pulse $A X 1$ is essentially the same pulse shape as the specularly reflected pulse. It is important to note that for both the spherical and cylindrical targets, the reference, specular, and back-axial pulses all have the same pulse shape to the accuracy of Fig. 4.

Utilizing $\mathrm{THz}$ impulse ranging to measure time resolved scattered electric fields, we have directly observed a manifestation of the Gouy phase shift. More importantly, this result has demonstrated the necessity to account for the single axis $\pi / 2$ phase shift when using a geometrical or physical optics approach to understanding scattering phenomena. Clearly, the relative polarity between the specular reflection $S$ and the scattered back axial reflection $A X 1$ is a signature which can reveal the geometry of the scattering target in a phase sensitive system.

This work was supported in part by the National Science Foundation and by the Army Research Office.

${ }^{1}$ R. A. Cheville, R. W. McGowan, and D. Grischkowsky, IEEE Trans. Antennas Propag. 45, 1518 (1997).

${ }^{2}$ R. A. Cheville, R. W. McGowan, and D. Grischkowsky, Phys. Rev. Lett. 80, 269 (1998).

${ }^{3}$ A. E. Siegman, Lasers (University Science Books, Mill Valley, CA, 1986), p. 682.

${ }^{4}$ S. Feng, H. G. Winful, and R. W. Hellwarth, Opt. Lett. 23, 385 (1998).

${ }^{5}$ A. B. Ruffin, J. F. Whitaker, S. Feng, H. G. Winful, and J. V. Rudd, in Conference on Lasers and Electro-Optics, 1999, OSA Technical Digest Series (Optical Society of America, Washington, DC, 1999), pp. 396397; see also: A. B. Ruffin, J. V. Rudd, J. F. Whitaker, S. Feng, and H. G. Winful, Phys. Rev. Lett. 83, 3410 (1999).

${ }^{6}$ R. W. McGowan, R. A. Cheville, and D. Grischkowsky, in Ref. 5, pp. 374-375.

${ }^{7}$ D. E. Barrick, Radar Cross Section Handbook, edited by G. T. Ruck (Plenum, New York, 1970), Vol. 1.

${ }^{8}$ J. Rheinstein, IEEE Trans. Antennas Propag. 16, 89 (1968).

${ }^{9}$ R. G. Kouyoumjian, L. Peters, Jr., and D. T. Thomas, IEEE Trans. Antennas Propag. 11, 690 (1963). 